\title{
Planets in orbit around $\beta$ Pictoris formed the orbital architecture of planetesimal belts?
}

\author{
Hiroshi Kimura $^{1 *}$, Misato Fukagawa ${ }^{2 \dagger}$, Motohide Tamura ${ }^{3,4}$, Hiroshi Kobayashi ${ }^{1 \ddagger}$, \\ Tetsuo Yamamoto ${ }^{1}$, Miki Ishii ${ }^{5}$, and Hiroshi Suto ${ }^{3}$ \\ ${ }^{1}$ Institute of Low Temperature Science, Hokkaido University, Kita-ku Kita-19 Nishi-8, Sapporo 060-0819, Japan \\ ${ }^{2}$ Division of Particle and Astrophysical Sciences, Graduate School of Science, Nagoya University, \\ Furo-cho, Chikusa-ku, Nagoya 464-8602, Japan \\ ${ }^{3}$ National Astronomical Observatory of Japan, 2-21-1 Osawa, Mitaka, Tokyo 181-8588, Japan \\ ${ }^{4}$ Department of Astronomical Science, The Graduate University for Advanced Studies, \\ 2-21-1 Osawa, Mitaka, Tokyo 181-8588, Japan \\ ${ }^{5}$ Subaru Telescope, 650 N. A'ohoku Place, Hilo, HI 96720, USA
}

(Received July 30, 2008; Revised November 11, 2009; Accepted November 11, 2009; Online published February 12, 2010)

\begin{abstract}
We report near-infrared imaging observations of the $\beta$ Pic dust disk, from which we infer the orbital architecture of planetesimal belts that remain near mean motion resonances (MMRs) with a planet at 62 AU. Our results reveal that one of the previously identified planetesimal belts lies in the 2/3 MMR with the planet, similar to the resonant relation between Plutinos and Neptune. We suggest that all the previously reported planetesimal belts are located near the 2/3 MMRs of four planets whose spatial arrangements make a similar figure of Jupiter, Saturn, Uranus, and Neptune. This implies that the Solar System is a prototype of planetary systems around main-sequence stars in terms of planets' configuration, as expected from planet formation theories.
\end{abstract}

Key words: Stars: individual ( $\beta$ Pictoris), stars: planetary systems, planetary systems: formation, Kuiper Belt, meteors, meteoroids.

\section{Introduction}

Imaging observations of the A5 $\mathrm{V}$ star $\beta$ Pictoris have revealed its circumstellar dust disk viewed almost edgeon from the Earth (Smith and Terrile, 1984; Paresce and Burrows, 1987; Golimowski et al., 1993, 2006; Kalas and Jewitt, 1995; Tamura et al., 2006). The disk is not primordial, but a dynamical consequence of debris that are continuously replenished by mutual collisions between planetesimals (Weissman, 1984; Backman et al., 1992; Backman and Paresce, 1993; Krivova et al., 2000). The location of the debris or dust is most likely confined near its parentbody planetesimals in nearly coplanar orbits (Krivov et al., 2000; Freistetter et al., 2007). This indicates that the spatial distribution of planetesimals can be traced through observed brightness profiles of the dust disk. A warp and rings in the inner disk suggest that the structures are formed

${ }^{*}$ Current affiliation: Center for Planetary Science, c/o Graduate School of Science of Kobe University, Nada-ku Rokkodai-cho 1-1, Kobe 6578501, Japan.

$\dagger$ Current affiliation: Department of Earth and Space Science, Graduate School of Science, Osaka University, 1-1 Machikaneyama, Toyonaka, Osaka 560-0043, Japan.

${ }^{\ddagger}$ Current affiliation: Astrophysical Institute and University Observatory, Friedrich Schiller University, Schillergaesschen 2-3, 07745 Jena, Germany.

Copyright (c) The Society of Geomagnetism and Earth, Planetary and Space Sciences (SGEPSS); The Seismological Society of Japan; The Volcanological Society of Japan; The Geodetic Society of Japan; The Japanese Society for Planetary Sciences; TERRAPUB.

doi:10.5047/eps.2009.11.001 by gravitational influences of planets on the planetesimals (Heap et al., 2000; Wahhaj et al., 2003; Okamoto et al., 2004; Freistetter et al., 2007). Surface brightness along the mid-plane of the $\beta$ Pic disk shows a kink in its radial profile around $100 \mathrm{AU}$ from the star (Golimowski et al., 1993, 2006; Kalas and Jewitt, 1995; Krivova et al., 2000). Such a brightness profile is consistent with the radial dust distribution that peaks around $100 \mathrm{AU}$, analogous to the dust in the Kuiper Belt (Backman et al., 1992; Backman and Paresce, 1993). These observations are well modeled under the assumption that planetesimals are concentrated in the range of 80-120 AU (Augereau et al., 2001; Thébault and Augereau, 2005). It is worth noting that, in the solar system, a number of trans-neptunian objects in the Kuiper Belt are trapped in mean motion resonances (MMRs) with Neptune (Luu and Jewitt, 2002). Analogous to the outer edge of the Kuiper Belt at the 1/2 MMR with Neptune, the sharp drop in the surface density of $\beta$ Pic planetesimals may be linked to the outer edge of a planetesimal belt at the $1 / 2 \mathrm{MMR}$ with a yet unseen planet, which is hereafter referred to as Planet $\mathrm{N}$. Near-infrared observations of linear polarization revealed a dip at 5.3 \pm 0.1 arcsec (i.e., 102-104 AU) in the north-east and south-west directions of the $\beta$ Pic disk (Tamura et al., 2006). We can interpret this dip as a paucity of planetesimals in the region from $1 / 2 \mathrm{MMR}$ to $4 / 9 \mathrm{MMR}$ with Planet N. Placing the dip in the middle of the $1 / 2$ and 4/9 MMRs, we would find Planet $\mathrm{N}$ at $62 \mathrm{AU}$ from the central star.

Spatially localized enhancements in the $K$-band brightness of the disk manifested a sign of multiple planetesimal rings (Tamura et al., 2006). If planetesimals in the $\beta$ Pic 
system reside in MMRs with a planet, the observed clumps in the surface brightness profile of the disk can be simply resonant structures of planetesimal belts. Therefore, we seek evidence for the presence of Planet $\mathrm{N}$ by investigating whether its MMRs match the locations of observed humps in the brightness distribution of the disk.

\section{Observation and Data Analysis}

Using the Subaru 8.2-m telescope and the CIAO infrared coronagraph imager with adaptive optics, we imaged the circumstellar dust disk of $\beta$ Pic in $H(1.65 \mu \mathrm{m})$ and $K$ $(2.2 \mu \mathrm{m})$ bands on January 4,2003 . A detailed description of the observations and the data reduction as well as a preliminary analysis of the $K$-band data can be found in Tamura et al. (2006). Hereafter, we focus on our data analyses to find observational evidence of multiple planetesimal rings in our data set. Signals of planetesimal rings would appear as brightness peaks in both northeast and southwest directions as well as both $H$ - and $K$-bands. We compare the excess emission in both bands after subtracting their continuum levels (see Section 3). A weak correlation with a correlation coefficient $(r)$ of 0.3 is indeed found between the clumps in $H$ - and $K$-bands. We assess the statistical significance of brightness peaks by testing the null hypothesis that the observed brightness distribution is merely random noise superimposed on a smooth curve of radiation scattered by circumstellar dust. Therefore, the random noise is smoothed out by averaging over both wavelengths and offset directions, resulting in a smooth declining curve of the average surface intensity. On the one hand, we admit that fake features might appear in the average brightness, if the random noise was not completely smoothed out by the averaging procedure. On the other hand, even real enhancements in the $K$-band brightness might be diluted with the $H$-band brightness, because the latter had a lower signalto-noise ratio than the former. In such an analysis, only strong peaks, which we attribute to nearly circular, coplanar rings, will remain in the residuals after subtraction of a smooth brightness component. Therefore, we derive the residuals from the mean of the surface intensities to trace possible resonant structures of multiple planetesimal rings associated with Planet N.

We use a dip at $5.3 \operatorname{arcsec}$ in the polarimetric data of Tamura et al. (2006) to infer the location of Planet $\mathrm{N}$ as analogous to the outer edge of the Kuiper Belt in the solar system, while the evolutionary history of the $\beta$ Pic system is likely different from that of the solar system. However, a different evolutional history does not necessarily mean that there is no paucity of planetesimals between the $1 / 2$ and $4 / 9$ MMRs of a planet in the $\beta$ Pic system. Therefore, we do not argue the presence of the planet at $62 \mathrm{AU}$ from an evolutional point of view, but we hereafter examine it with our observational data. Note that the dip at 5.3 arcsec in our polarimetric data is simply our motivation to seek a planet around $62 \mathrm{AU}$.

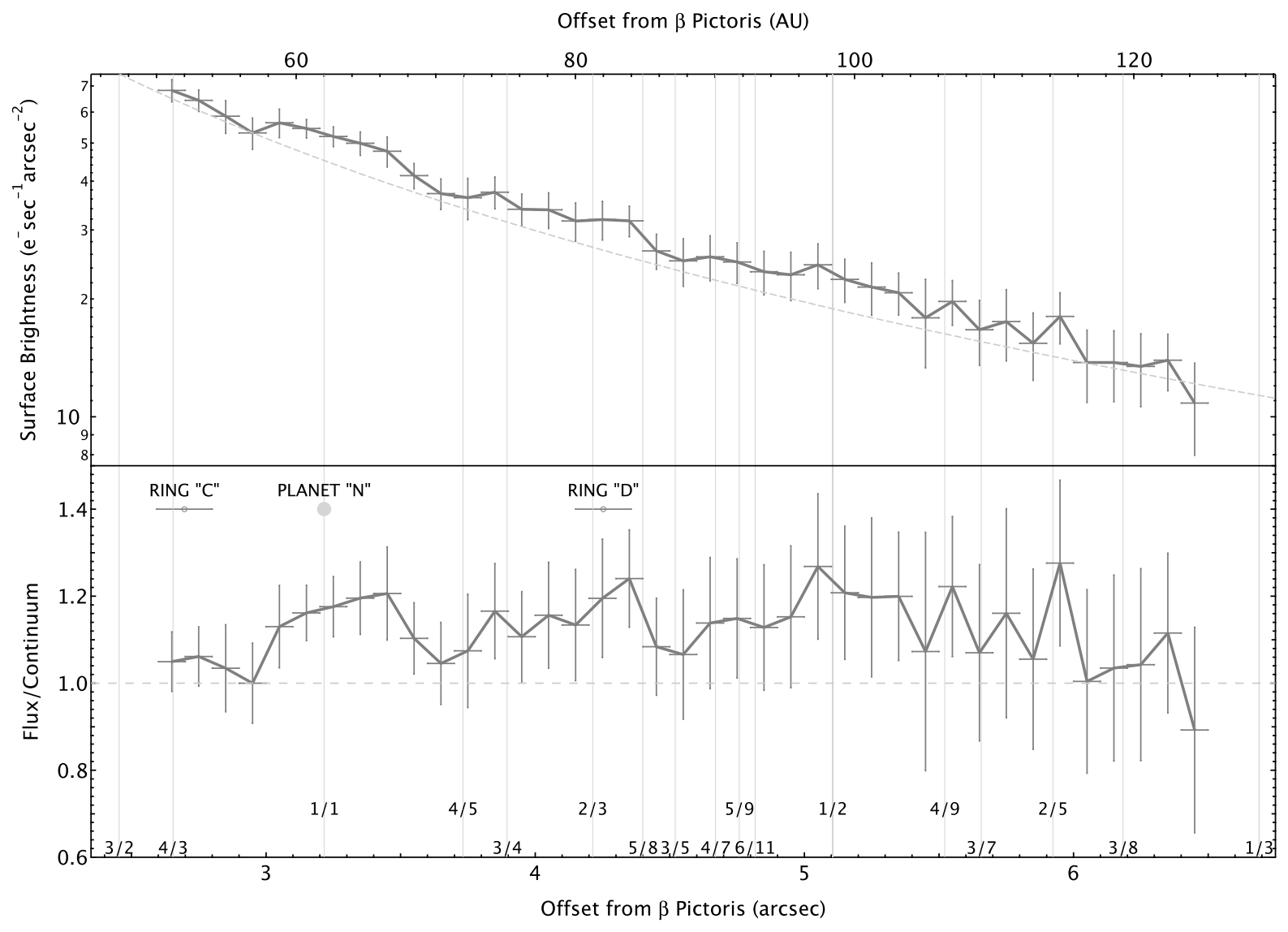

Fig. 1. Resonance structures in the composite near-infrared intensity of the dust disk around $\beta$ Pictoris. Also indicated are the locations of possible planetesimal belts, Ring C and Ring D found by Wahhaj et al. (2003), and the outermost planet, called Planet N. 


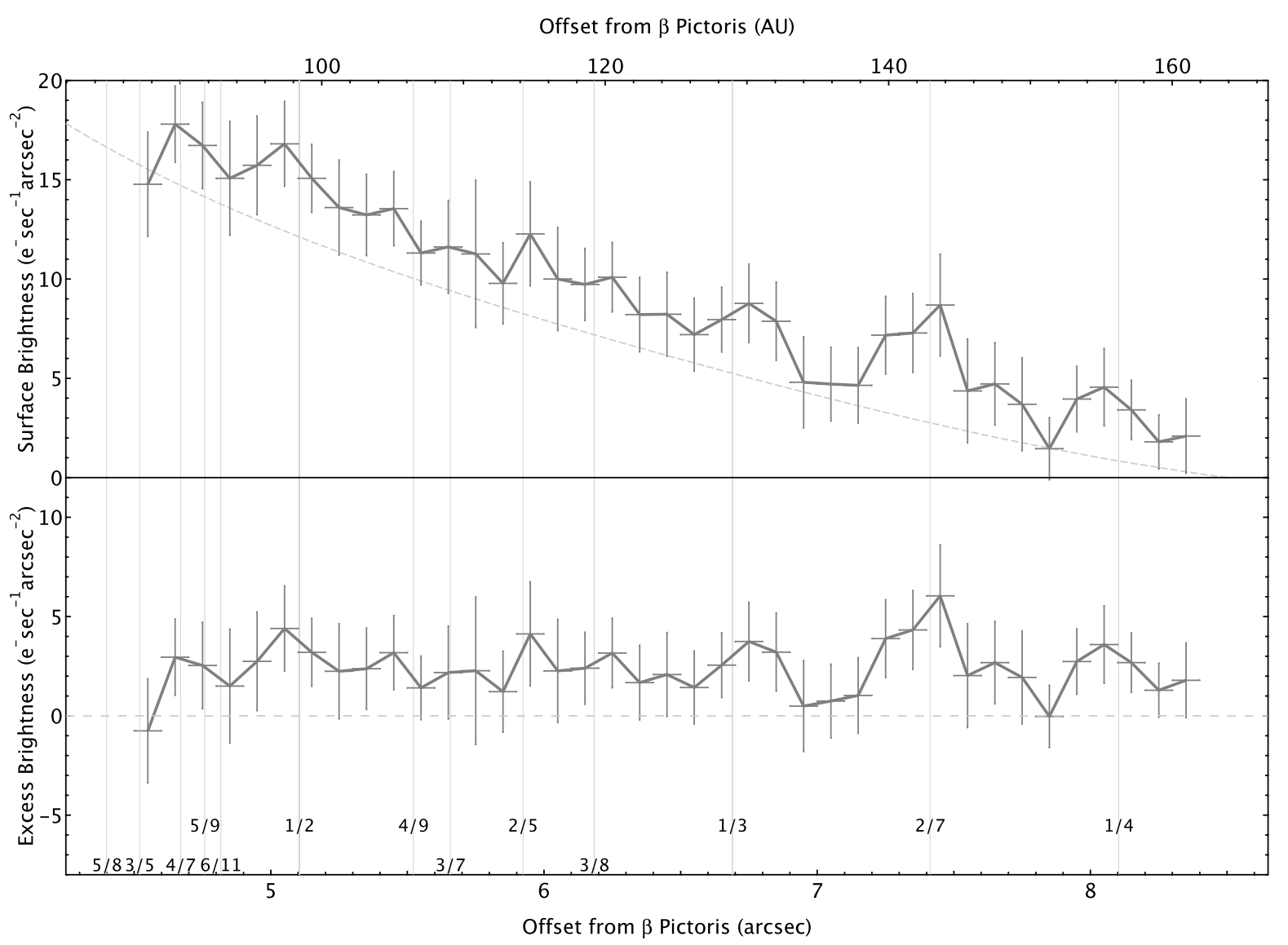

Fig. 2. Resonance structures in the $K$-band intensity of the dust disk around $\beta$ Pictoris.

\section{Results}

Figure 1 shows the geometric mean of the four surface intensities and a smooth declining curve, "continuum", as a function of radial distance from the central star. Here we take a smooth declining curve at the lowest envelope of the data as being the continuum, which is the sum of real smooth brightness and true random noise, since the random noise has an additive effect. Also plotted is the ratio of the geometric mean flux to the continuum, which gives a measure of excess intensities (clumps). If the null hypothesis is true, we would expect that any peculiar features cancel out by the averaging over two directions and two wavelengths. The vertical lines indicate the locations of the MMRs associated with Planet $\mathrm{N}$ in orbit around $\beta$ Pic at $62 \mathrm{AU}$. We find $2 \sigma$ excess above the continuum brightness around the 1/1 and 2/3 MMRs with Planet $\mathrm{N}$ at $62 \mathrm{AU}$. Given this result, we would be inclined to reject the null hypothesis and to accept the alternative hypothesis that the $2 \sigma$ excess indicates real features of planetesimal rings. If we regard $1 \sigma$ excess over three bins to be a real feature, an additional feature appears in the $1 / 2 \mathrm{MMR}$. We realize the presence of similar enhancements around $1 / 2$ and 2/3 MMRs in the $K$-band brightness measured by Mouillet et al. (1997). It is worth noting that the D ring identified by Wahhaj et al. (2003) in their mid-infrared images lies in the 2/3 MMR with Planet N. Taking into account the occurrence of the 2/3 MMR peak in three independent infrared observations, it is unlikely that the null hypothesis is true. The correlation between the MMR and the clump locations is also inferred by $r=0.3$, which supports our assessment.

Identification of MMRs at larger distances is easier because of a wider interval between two neighboring MMRs beyond the 1/2 MMR (i.e., 5 arcsec). Unfortunately the $H$-band beyond 6 arcsec intensity suffers from low signalto-noise ratios and its radial slope departs from that of the $K$-band intensity. Therefore, we use only the $K$-band data to identify further clumps of planetesimals in the surface brightness beyond 5 arcsec. Figure 2 shows the structures of clumps in the $K$-band surface brightness derived from the arithmetic mean of the north-east and south-west intensities. The positions of brightness humps appear to coincide with the locations of MMRs, in particular, 1/2, 1/3, 2/7 and $1 / 4$. We find that the correlation of locations between these MMRs and the observed clumps is stronger $(r=0.4)$ than in the inner region. In this distance range, the intervals of MMRs are greater than the FWHM of approximately 0.19 arcsec in the $K$-band. Accordingly, it is unlikely that the locations of the residual clumps and the MMRs overlap by chance. Contrary to the null hypothesis, this gives indirect evidence for the presence of Planet $\mathrm{N}$ at $62 \mathrm{AU}$.

\section{Discussion}

\subsection{Large dust particles}

The radial distribution of edge-on-disk brightness traces the radial distribution of the dust that dominates the total cross section of light scattering by dust particles in the disk. If the size distribution of the dust in the $\beta$ Pic disk is similar to that of interplanetary dust in the solar system or that 
of cometary dust, the scattering cross section is determined by large dust particles (Grün et al., 1985; McDonnell et al., 1987; Kolokolova et al., 2007). Indeed, the infrared spectra of cometary dust and $\beta$ Pic dust are very much alike (Knacke et al., 1993). Although this does not guarantee the similarity in the size distribution between cometary dust and $\beta$ Pic dust, we do not find a reason that small dust particles dominate the scattering cross section. Large dust particles tend to scatter stellar radiation in forward directions and to stay near the orbit of their parent bodies (e.g., Kresák,1976; Bohren and Huffman, 1983). Polarimetric observations of the $\beta$ Pic debris disk have revealed an effect of strong forward scattering that decreases the degree of linear polarization with decreasing radial distance from the star (Krivova et al., 2000; Tamura et al., 2006). The strong forward scattering is also manifested in the pronounced dip around 5.3 arcsec in the radial profile of linear polarization, in contrast to its insignificant correspondence in the radial profile of our $K$-band intensity (Tamura et al., 2006). Since large dust particles are less affected by stellar radiation pressure, they accumulate along the orbit of their parent body as seen in dust trails of comets (e.g., Ishiguro et al., 2002). In addition, collisional models of debris disks predict that the peak of thermal radiation from dust particles appears at a planetesimal belt in a typical debris disk (Krivov et al., 2008). Even though the location of parent-body planetesimals may not exactly coincide with associated dust rings, the difference is not necessarily notified with our spacial resolution of approximately $4 \mathrm{AU}$. Therefore, the location of a planetesimal belt can be identified by observations of its associated dust ring, unless the $\beta$ Pic dust is very different from the dust in the solar system and in typical debris disks. We conclude that observations of stellar radiation scattered by dust particles in a typical debris disk would provide an opportunity of detecting extrasolar planets in the disk if the orbital architecture of planetesimal belts are shaped by the gravitational field of the planets.

\subsection{The outermost planet}

Even though the radial distribution of planetesimals steeply drops near the $1 / 2 \mathrm{MMR}$ with Planet $\mathrm{N}$, the $\beta$ Pic system appears to consist of planetesimal rings on the outskirts of the planetesimal disk beyond the 1/2 MMR. We note that we certainly have to wait for future observations to confirm our identification with these high-order resonances, which could be criticized to be premature at this stage. Hahn and Malhotra (1999) have shown that planetary migration results in concentration of planetesimals at the outermost planet's exterior MMRs and allows the planetesimals beyond 1/2 MMR to remain in orbits with low eccentricities and low inclinations. The resonant planetesimals beyond Planet N's 1/2 MMR seem to reside in nearly circular, low-inclination orbits, because they are identified at the same distance from the star along northeast and southwest directions. This may indicate that these outer planetesimal rings around $\beta$ Pic are relatively undisturbed remnants of the natal planetesimal disk. Although the numerical results by Hahn and Malhotra (1999) might depend on various input parameters, we may expect that Planet $\mathrm{N}$ is the outermost planet in the $\beta$ Pic system.

Owing to its spatially resolved edge-on viewing, the width of the $\beta$ Pic disk has been measured over a wide range of distances. The disk width increases with distance in the outer disk ( $>100 \mathrm{AU})$, but it is almost constant in the inner disk (<100 AU) (Kalas and Jewitt, 1995; Krivova et al., 2000; Golimowski et al., 2006; Tamura et al., 2006). The flared structure of the outer disk is akin to the structure of the outer Kuiper Belt where the planetesimal disk is flared up from the ecliptic plane. Lecavelier des Etangs (1998) pointed out that the vertical structure of the $\beta$ Pic disk might have resulted from the outward migration of multiple giant planets. In terms of Planet $\mathrm{N}$, planetary migration is consistent with a planet formation scenario that precludes insitu formation of a planet at $62 \mathrm{AU}$ around $\beta$ Pic (Nakano, 1988). To place a planet at $62 \mathrm{AU}$ around $\beta$ Pic, however, the planet formation scenario might require rapid planet migration that is at odds with nearly circular, coplanar planetesimal rings. Consequently, the presence of Planet $\mathrm{N}$ at 62 AU would give new insights into formation of a planetary system, in particular, planetary migration.

\subsection{Planetary system}

Mid-infrared images of the inner $\beta$ Pic disk revealed four inner rings at $14 \pm 1$ (A ring), $28 \pm 3$ (B ring), $52 \pm 2$ (C ring), and $82 \pm 2 \mathrm{AU}$ (D ring) with the greatest optical depth in the D ring (Wahhaj et al., 2003). We notice that the location of the D ring corresponds to the 2/3 MMR of planetesimals with Planet $\mathrm{N}$ at 81.2 AU. Note that Pluto and Plutinos in the $2 / 3 \mathrm{MMR}$ with the outermost planet Neptune are known to have stable orbits (Luu and Jewitt, 2002). Therefore, the greatest optical depth of the D ring is reasonably a consequence of the strong $2 / 3 \mathrm{MMR}$ with Planet $\mathrm{N}$, the outermost planet of the $\beta$ Pic system. In addition to the $\mathrm{A}$ and $\mathrm{B}$ rings, mid-infrared spectral features of silicates revealed the presence of a planetesimal belt $(\mathrm{O}$ ring) around $6 \mathrm{AU}$ (Okamoto et al., 2004). If the $\mathrm{O}$ ring were a main asteroid belt in relation to a yet undetected planet having the A ring in its 2/3 MMR, the hypothetical planet, which is hereafter referred to as Planet $\mathrm{J}$, would exist at 11 AU. In fact, Jupiter-mass Planet J around 10-12 AU in a slightly eccentric orbit is an appropriate solution to generate the warp of the disk and a number of star-grazing comets observed in the $\beta$ Pic system (Lecavelier des Etangs, 1998; Heap et al., 2000; Freistetter et al., 2007). Located slightly inside Planet $\mathbf{J}$ is the ice boundary around $9 \mathrm{AU}$ from the central star. A model of planet formation indicates that gas giants can form slightly outside the ice boundary (Ida and Lin, 2004). Therefore, we expect Planet J, if exists, to be a gas giant ${ }^{1}$.

The slightly eccentric orbit of Planet $\mathbf{J}$ may indicate the presence of another external giant planet, which is hereafter termed Planet S (Thébault and Beust, 2001). If the near $5 / 2$ resonant relation between Jupiter and Saturn in the Solar System holds in the $\beta$ Pic system, one may find Planet $\mathrm{S}$ orbiting $\beta \mathrm{Pic}$ at $20 \mathrm{AU}$. Interestingly, the $2 / 3 \mathrm{MMR}$ with Planet $\mathrm{S}$ is located at 26.2 AU where the B ring was identified by infrared observations. Furthermore, we realize

\footnotetext{
${ }^{1}$ When revising the manuscript, Lagrange et al. (2009a, b) reported a probable detection of a giant planet around $8 \mathrm{AU}$, which is in accord with the location of Planets J within the accuracy of the observational data used
} in our discussion. 


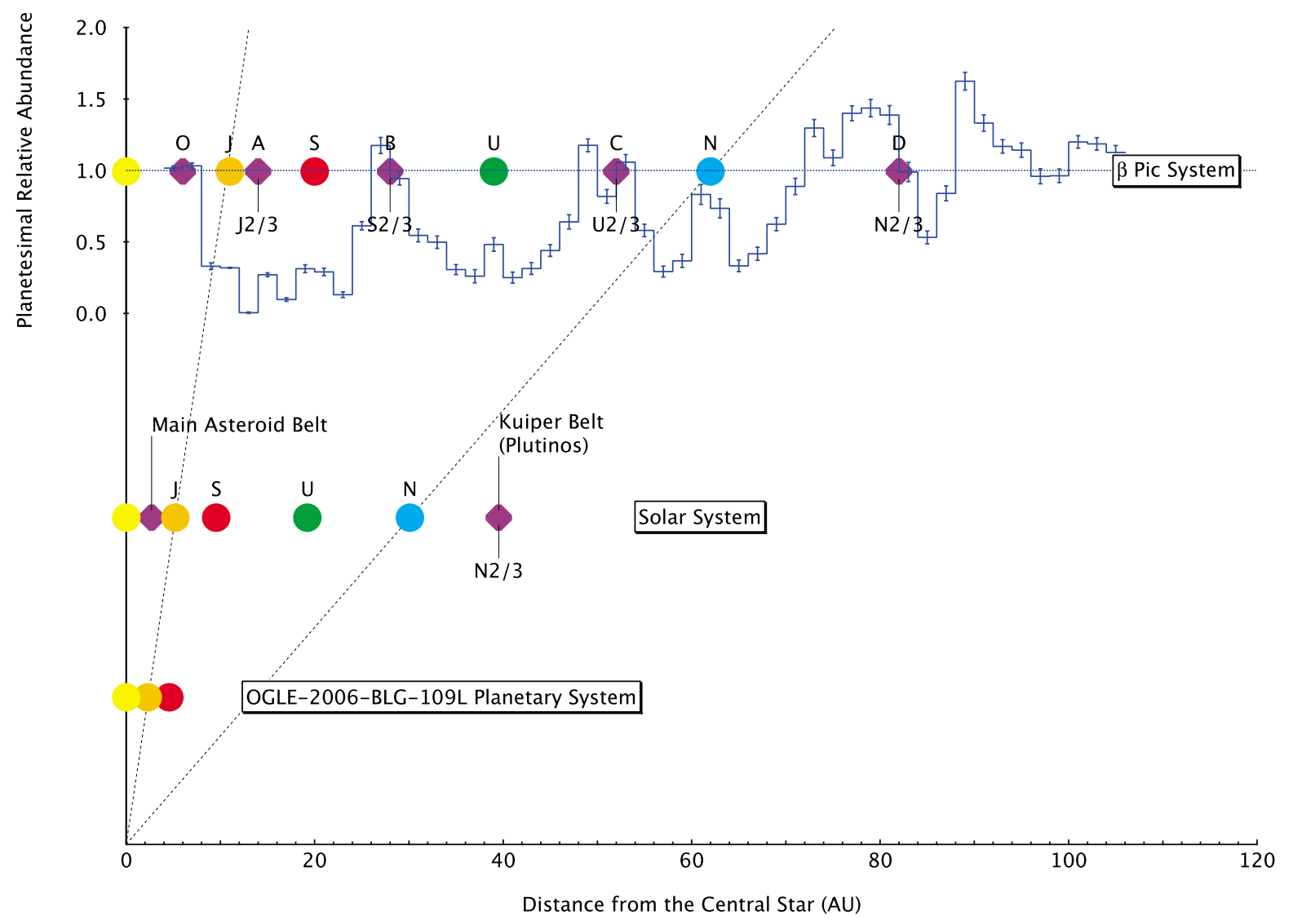

Fig. 3. Illustration of planetary systems around $\beta$ Pic, the Sun, and OGLE-2006-BLG-109L. The extent of the $\beta$ Pic system is twice as large as that of the Solar System. In the $\beta$ Pic system, the A, B, C, and D rings are associated with the $2 / 3$ mean-motion resonance of Planets J, S, U, and N, while the $\mathrm{O}$ ring corresponds to the main asteroid belt inside the ice boundary.

that the semimajor axis ratio of Planets $\mathrm{J}$ and $\mathrm{N}$ is similar to that of Jupiter and Neptune. This leads us to speculate the presence of a planetary system in the $\beta$ Pic disk that is akin to our own planetary system. If the near $2 / 1$ resonant relation between Neptune and Uranus also holds in the $\beta$ Pic system, a planet, which we call Planet $\mathrm{U}$, would orbit $\beta$ Pic at $39 \mathrm{AU}$ with the $\mathrm{C}$ ring in the $2 / 3 \mathrm{MMR}$ at 51.1 AU. In Fig. 3, we provide our perspective picture of the planetary system around $\beta$ Pic together with the Solar System and the OGLE-2006-BLG-109L planetary system (see Gaudi et al., 2008). One could find that Planets J, S, $\mathrm{U}$ and $\mathrm{N}$ are all accompanied with a planetesimal ring in their $2 / 3$ MMRs. A very recent numerical study on the dynamical evolution of planetesimals in the $\beta$ Pic system with three planets suggests the presence of a Saturn-mass planet around $25 \mathrm{AU}$ and a Uranus-mass planet around $44 \mathrm{AU}$ in addition to a Jupiter-mass planet around $12 \mathrm{AU}$ (Freistetter et al., 2007). We have also performed a similar dynamical simulation of planetesimals with our four planets J, S, U and $\mathrm{N}$ in coplanar circular orbits at their expected locations. ${ }^{2}$ The fourth-order predictor-corrector method is used for the

\footnotetext{
${ }^{2}$ We place 2, 0.5, 0.1, 0.1 Jovian-mass planets to simulate their gravitational influences on planetesimals initially distributed from 5 to $110 \mathrm{AU}$ in a disk according to a power-law radial distribution with the power of -0.5 . Our results on the relative abundance of planetesimals do not depend on the assumed distribution of planetesimals, because each test particle in our simulation has no gravitational force on the other particles.
}

numerical simulation on the dynamical evolution of planetesimals over 12 Myrs (see for the method Kokubo and Makino, 2004). Our results of numerical simulation indicate that planetesimals tend to stay in orbit near $1 / 1$ and $2 / 1$ MMRs of the planets. The masses of the planets would be constrained by further simulations, if we take into account the fact that our observational data do not show the secular resonance around $90 \mathrm{AU}$, which depends on the planets' masses. The numerical results appended in Fig. 3 are consistent not only with our perspective but also with observational results given in Fig. 1. By taking account of the observational uncertainties in the locations of the planetesimal rings, these numerical results support our perspective given in Fig. 3.

\section{Concluding Remarks}

The presence of a planetary system around $\beta$ Pic is consistent with available observations of the $\beta$ Pic dust disk from near- to mid-infrared wavelengths. The detection of planetesimal rings in the visible wavelength range is more difficult, because a number of clumps in the foreground more easily obscure a planetesimal ring that is tangential to the line of sight. In fact, optical observations with Hubble Space Telescope have not succeeded in imaging the clumps of planetesimals up to date (Golimowski et al., 2006). On the one hand, higher resolution infrared observations are able to reveal the resonant structures of planets and to identify their precise locations. On the other hand, numerical 
dynamical simulations of planetesimals in the stellar and planetary gravitational fields allow us to constrain the mass and orbital parameters of the planets. If the presence of Planets J, S, U and $\mathrm{N}$ were confirmed by future observations, then the coming question would be: Are there Earthlike planets in the $\beta$ Pic system? A radial velocity survey excludes the presence of an inner giant planet in the immediate vicinity of the star ( $\leq 1 \mathrm{AU})$ (Galland et al., 2006). However, the result does not contradict the presence of Earth-like planets in orbit around $\beta$ Pic within a few $\mathrm{AU}$ from the star.

Acknowledgments. This research is supported by grants from CPS, JSPS, and MEXT Japan.

\section{References}

Augereau, J. C., R. P. Nelson, A. M. Lagrange, J. C. B. Papaloizou, and D. Mouillet, Dynamical modeling of large scale asymmetries in the $\beta$ Pictoris dust disk, Astron. Astrophys., 370, 447-455, 2001.

Backman, D. E. and F. Paresce, Main-sequence stars with circumstellar solid material: the Vega phenomenon, in Protostars and Planets III, edited by E. H. Levy and J. I. Lunine, pp. 1253-1304, Univ. Arizona Press, Tucson, 1993.

Backman, D. E., F. C. Gillett, and F. C. Witteborn, Infrared observations and thermal models of the $\beta$ Pictors disk, Astrophys. J., 385, 670-679, 1992.

Bohren, C. F. and D. R. Huffman, Absorption and Scattering of Light by Small Particles, Wiley-Interscience, New York, 1983.

Freistetter, F., A. V. Krivov, and T. Löhne, Planets of $\beta$ Pictoris revisited, Astron. Astrophys., 466, 389-393, 2007.

Galland, F., A.-M. Lagrange, S. Udry, A. Chelli, F. Pepe, J.-L. Beuzit, and M. Mayor, Extrasolar planets and brown dwarfs around A-F type stars. III. $\beta$ Pictoris: looking for planets, finding pulsations, Astron. Astrophys., 447, 355-359, 2006.

Gaudi, B. S. et al., Discovery of a Jupiter/Saturn analog with gravitational microlensing, Science, 319, 927-930, 2008.

Golimowski, D. A., S. T. Durrance, and M. Clampin, Coronagraphic imaging of the $\beta$ Pictoris circumstellar disk: Evidence of changing disk structure within $100 \mathrm{AU}$, Astrophys. J., 411, L41-L44, 1993.

Golimowski, D. A. et al., Hubble Space Telescope ACS multiband coronagraphic imaging of the debris disk around $\beta$ Pictoris, Astron. J., 131, 3109-3130, 2006

Grün, E., H. A. Zook, H. Fechtig, and R. H. Giese, Collisional balance of the meteoritic complex, Icarus, 62, 244-272, 1985.

Hahn, J. M. and R. Malhotra, Orbital evolution of planets embedded in a planetesimal disk, Astron. J., 117, 3041-3053, 1999.

Heap, S. R., D. J. Lindler, T. M. Lanz, R. H. Cornett, I. Hubeny, S. P. Martin, and B. Woodgatte, Space telescope imaging spectrograph coronagraphic observations of $\beta$ Pictoris, Astrophys. J., 539, 435-444, 2000.

Ida, S. and D. N. C. Lin, Toward a deterministric model of planetary formation. I. A desert in the mass and semimajor axis distributions of extrasolar planets, Astrophys. J., 604, 388-413, 2004.

Ishiguro, M., J. Watanabe, F. Usui, T. Tanigawa, D. Kinoshita, J. Suzuki, R. Nakamura, M. Ueno, and T. Mukai, First detection of an optical dust trail along the orbit of 22P/Kopff, Astrophys. J., 572, L117-L120, 2002.

Kalas, P. and D. Jewitt, Asymmetries in the Beta Pictors dust disk, Astron. J., 110, 794-804, 1995.
Knacke, R. F., S. B. Fajardo-Acosta, C. M. Telesco, J. A. Hackwell, D. $\mathrm{K}$. Lynch, and R. W. Russell, The silicates in the disk of $\beta$ Pictoris, Astrophys. J., 418, 440-450, 1993.

Kokubo, E. and J. Makino, A modified Hermite integrator for planetary dynamics, Publ. Astron. Soc. Jpn., 56, 861-868, 2004.

Kolokolova, L., H. Kimura, N. Kiselev, and V. Rosenbush, Two different evolutionary types of comets proved by polarimetric and infrared properties of their dust, Astron. Astrophys., 463, 1189-1196, 2007.

Kresák, L., Orbital evolution of the dust streams released from comets, Bull. Astr. Inst. Czechosl., 27, 35-46, 1976.

Krivov, A. V., I. Mann, and N. A. Krivova, Size distributions of dust in circumstellar debris discs, Astron. Astrophys., 362, 1127-1137, 2000.

Krivov, A. V., S. Müller, T. Löhne, and H. Mutschke, Collisional and thermal emission models of debris disks: Toward planetesimal population properties, Astrophys. J., 687, 608-622, 2008.

Krivova, N. A., A. V. Krivov, and I. Mann, The disk of $\beta$ Pictoris in the light of polarimetric data, Astrophys. J., 539, 424-434, 2000.

Lagrange, A.-M. et al., A probable giant planet imaged in the $\beta$ Pictoris disk. VLT/NaCo deep L'-band imaging, Astron. Astrophys., 493, L21L25, 2009a.

Lagrange, A.-M. et al., Constraining the orbit of the possible companion to $\beta$ Pictoris. New deep imaging observations, Astron. Astrophys., 506, 927-934, 2009b.

Lecavelier des Etangs, A., Planetary migration and sources of dust in the $\beta$ Pictoris disk, Astron. Astrophys., 337, 501-511, 1998.

Luu, J. X. and D. C. Jewitt, Kuiper Belt Objects: Relics from the accretion disk of the Sun, Ann. Rev. Astron. Astrophys., 40, 63-101, 2002.

McDonnell, J. A. M. et al., The dust distribution within the inner coma of comet P/Halley 1982i: encounter by Giotto's impact detectors, Astron. Astrophys., 187, 719-741, 1987.

Mouillet, D., A.-M. Lagrange, J.-L. Beuzit, and N. Renaud, A stellar coronagraph for the COME-ON-PLUS adaptive optics system. II. First astronomical results, Astron. Astrophys., 324, 1083-1090, 1997.

Nakano, T., Formation of planets around stars of various masses II. Stars of two and three solar masses and the origin and evolution of circumstellar dust clouds, Mon. Not. Roy. Astron. Soc., 230, 551-571, 1988.

Okamoto, Y. K. et al., An early extrasolar planetary system revealed by planetesimal belts in $\beta$ Pictoris, Nature, 431, 660-663, 2004.

Paresce, F. and C. Burrows, Broad-band imaging of the Beta Pictoris circumstellar disk, Astrophys. J., 319, L23-L25, 1987.

Smith, B. A. and R. J. Terrile, A circumstellar disk around $\beta$ Pictoris, Science, 226, 1421-1424, 1984.

Tamura, M., M. Fukagawa, H. Kimura, T. Yamamoto, H. Suto, and L. Abe, First two-micron imaging polarimetry of $\beta$ Pictoris, Astrophys. J., 641, 1172-1177, 2006.

Thébault, P. and J.-C. Augereau, Upper limit on the gas density in the $\beta$ Pictoris system: The effect of gas drag on dust dynamics, Astron. Astrophys., 437, 141-148, 2005.

Thébault, P. and H. Beust, Falling evaporating bodies in the $\beta$ Pictoris system. Resonance refilling and long term duration of the phenomenon, Astron. Astrophys., 376, 621-640, 2001.

Wahhaj, Z., D. W. Koerner, M. E. Ressler, M. W. Werner, D. E. Backman, and A. I. Sargent, The inner rings of $\beta$ Pictoris, Astrophys. J., 584, L27L31, 2003.

Weissman, P. R., The Vega particulate shell: comets or asteroids?, Science, 224, 987-989, 1984.

H. Kimura (e-mail: hiroshi@kitty.kobe-u.ac.jp), M. Fukagawa, M. Tamura, H. Kobayashi, T. Yamamoto, M. Ishii, and H. Suto 